Ann. Sci. forest., 1970, 27 (3), 323-327.

INFORMATIONS

\title{
GROUPE D'ÉTUDE DES PROBLÈMES DE PHYSIOLOGIE DE L'ARBRE
}

\author{
RÉSUMÉS DES COMMUNICATIONS PRÉSENTÉES A LA SÉANCE \\ DU 16 OCTOBRE 1969
}

\section{Jacquiot (Directeur de Recherches, Centre technique du Bois).}

Cultures de tissus d'ARbres Conservées aU laboratoire de biologie VÉGétale de FONTAINEBLEAU.

Des souches installées de plusieurs espèces d'arbres forestiers sont conservées depuis un assez grand nombre d'années ( 21 ans pour celle de Castanea vesca). Parmi ces souches, les unes peuvent être conservées sur un milieu contenant exclusivement des sels minéraux et un glucide (C. vesca Gaertn.) (Betula verrucosa Ehr.), d'autres (Populus tremula L.) (Prunus avium Moench.) exigent l'apport d'auxine, d'autres exigent un apport de cytokinine (Quercus sp.) ou de cytokinine et d'auxine (Fagus sylvatica L.). Le tissu d'Eucalyptus camaldulensis n'a pu être conservé gue sur un milieu au lait de coco. Les essais de dérivés de la $\sigma$-aminopurine ont montré que la $\sigma$-benzylaminopurine, la $\sigma$-méthyl et la $\sigma$-diméthylaminopurine manifestent vis-à-vis de divers tissus les propriétés de cytokinines; l'utilisation de substances de ce groupe ouvre done des perspectives nouvelles pour la culture indéfinie des tissus végétaux jusqu'ici réfractaires.

Les phénomènes d'organogenèse ont donné lieu à de nombreuses observations, en particulier sur la formation tardive de racines ne se manifestant qu'après un nombre élevé de repiquages.

R. Keller (I.N.R.A., Station de Recherches sur la Qualité des Bois, Nancy).

\section{A propos de l'élagage du PEUPLIER.}

Des mesures d'accroissement en circonférence et en hauteur effectuées dans un dispositif d'élagage de peupliers I, 214 plantés à $7 \mathrm{~m} \times 7 \mathrm{~m}$ dans la région de Villers-Cotterets ont montré que la réaction de cette essence dans la saison qui suit le traitement est différente de ce que l'on a pu observer jusqu'ici dans des expériences analogues portant sur des essences résineuses. 
Le dispositif est un carré latin où un tiers des peupliers furent élagués à $50 \%$ de leur hauteur totale initiale, un autre tiers à $60 \%$, le dernier tiers servant de témoins.

Alors que, dans les expériences d'élagage de résineux, la croissance en circonférence des arbres traités est inférieure à celle des témoins dans les parties basses des troncs, ou au plus égale dans la zone de la base de la cime vivante, chez le peuplier une nouveauté apparaît : si le phénomène dépressif se manifeste toujours à la base des arbres, il disparaît complètement dans la cime. Pour cette zone, ce sont en effet les arbres qui ont subi le taux d'élagage le plus fort qui présentent l'accroissement en circonférence le plus élevé. Leur croissance en hauteur est également la plus grande.

Il se pourrait que chez les témoins, la présence d'une grande surface foliaire favorise une évaporation intense difficilement compensée, à certains moments, par l'absorption de l'eau du sol et entraînant, par suite de la fermeture des stomates, l'arrêt de la photosynthèse qui toucherait en premier lieu la partie haute de l'arbre; les niveaux inférieurs étant plus proches du sol, moins ensoleillés et plus à l'abri du vent subiraient moins longtemps le phénomène, et la croissance y serait moins affectée.

Chez les arbres élagués, la réduction de la surface foliaire diminue l'évaporation et l'approvisionnement en eau risque moins d'être insuffisant. La photosynthèse pourrait donc être moins troublée dans les cimes qui auraient alors une croissance en diamètre et en hauteur supérieure à celle des témoins, comme le montrent les mesures. L'enlèvement des branches basses provoque sur les niveaux correspondants du tronc un phénomène dépressif classique.

Une étude approfondie de ces phénomènes devrait probablement tenir compte des types d'activités photosynthétiques des feuilles aux différents niveaux, comparer la densité des stomates sur les feuilles des témoins et des arbres traités, étudier la répartition de l'auxine.

Quoi qu'il en soit, des observations ultérieures montrent que les faits observés ne durent qu'une saison de végétation, celle qui suit l'élagage.

\section{Roussel (Ingénieur en chef des Eaux et Forêts)}

RÉALISATION D’UN PETIT SIMULATEUR DE PHOTOSYNTHÈSE.

L'auteur présente un appareil très simple, dont l'élément essentiel est une cellule photorésistante au sulfure de cadmium activé, alimentée par une pile ordinaire de 4,5 volts, et actionnant un micromoteur électrique. Les constituants sont choisis de telle sorte que la vitesse du micromoteur, variant selon la lumière reçue par la cellule, reproduit assez bien l'allure de l'intensité de la photosynthèse d'une feuille de hêtre, en fonction de la même lumière.

\section{Totalisateur de Lumière Électronique autonome.}

L'auteur présente également un totalisateur de lumière électronique, qui, placé en un lieu quelconque, indique le nombre de lux/heures reçus par l'appareil, pendant une période de temps déterminée. L'autonomie actuelle est de 1 à 2 mois avant la recharge, peu coûteuse, de l'appareil qui peut être utilisé en physiologie végétale, écologie, etc...

\section{Autres communications.}

Nouvelles expériences sur «l'effet manchon», chez 6 espèces résineuses (d'ombre et de lumière), dont les graines ont été fournies par le C.N.R.F. Retour exceptionnel (et léger) au 
phototropisme juvénile chez quelques résineux, mis en conditions de souffrance et en lumière fortement déséquilibrée. Bilan de 8 années d'observations sur 12 espèces résineuses, d'origine contrôlée, soumises à 6 rayonnements relatifs différents dans le milieu forestier. Nouveaux essais (non encore concluants) sur la modification du pouvoir réflecteur des tiges et des feuilles du chêne rouge d'Amérique, et sur la localisation de l'effet manchon.

\section{B. Boullard (Biologie végétale, Faculté des Sciences de Rouen, 76 - Mont-St-Aignan).}

\section{Remarques Sur le Problème de l’Armillaire dans les peuplements de résineuX EXotiques en HaUte-Normandie.}

Les forêts hauts-normandes, traditionnellement vouées en grande majorité aux feuillus, sont en pleine mutation. Des impératifs d'ordre économique d'une part, la spectaculaire réussite d'Emile Maire en forêt d'Eu, au début du siècle d'autre part, ont en effet «relancé » l'intérêt des essences résineuses. L'Armillaire [Armillariella mellea (Vahl.) Karst.], champignon très commun, mais pratiquement inoffensif dans les peuplements feuillus, se révèle de jour en jour plus redoutable à l'encontre des Sitka, Douglas et autres Grandis.

Des conditions climatiques particulières sont peut-être responsables de ce fâcheux réveil depuis quelques années. Mais le biologiste ne peut s'empêcher de penser que l'installation de vastes surfaces monospécifiques d'essences exotiques y soit aussi pour quelque chose. Les caractères morphologiques et biologiques du Champignon étant réévoqués, l'auteur met ensuite le reboiseur en garde contre une mode de l'enrésinement généralisé dont il pourrait se repentir à assez bref délai.

\section{J. Crabbé (C.N.E.C.F., Gembloux, Belgique).}

\section{LOCALISATION ET CARACTÈres DEs RAMifications SUSCITÉEs PAR L'ARCURE DE LA} POUSSE D'UN AN DE BOUTURES DE POMMIER.

Le but de l'essai présenté était de décrire avec précision la réaction à l'arcure pour une forme-type d'arc et un matériel vigoureux, en l'occurrence des boutures de pommier Malling Merton 104.

Au mois de janvier, la moitié supérieure de l'axe de ces plantes a été arquée de manière que l'apex soit dirigé verticalement vers le sol. Les ramifications latérales formées par suite de ce traitement, réparties en catégories différentes selon leur vigueur, sont dénombrées dans les diverses zones du plant arqué. Sur la moitié des plants, un traitement supplémentaire supprime, à la fin mai, toutes les pousses ayant commencé de s'allonger.

L'analyse des fréquences d'apparition des divers types de ramifications aux différents nœuds de l'axe arqué permet de définir un «modèle» de réaction. La réalité de ce «modèle» est attestée par sa restauration approximative après la profonde perturbation apportée par les suppressions de fin mai.

Ce «modèle» est caractérisé par l'existence d’un groupe de pousses dominant, composé en moyenne de 2 pousses très vigoureuses et de 1 à 2 de vigueur moyenne. La ramification la plus vigoureuse de ce groupe occupe une position proche du sommet de l'arc, sur la face dorsale et au plus près du plan de symétrie du rameau arqué. La composition et la disposition du groupe dominant, par rapport à la pousse la plus vigoureuse, sont commandées par la phyllotaxie du rameau arqué. 


\section{H. Lakhoua (Gembloux, Belgique).}

\section{MODIFICATIONS APPORTÉES A LA RÉACTION A L'ARCURE PAR DIVERS DEGRÉS D'INCLI- NAISON DE LA PORTION APICALE DE LA POUSSE D'UN AN CHEZ LE POMMIER.}

Le présent travail étudie les réactions du rameau d'un an de marcottes de pommier E.M. VII âgées de deux ans, ayant subi trois formes d'arcure réalisées en février 1969. Selon que la partie terminale du rameau est dirigée vers le bas, horizontalement ou obliquement vers le haut, on a une arcure complète (A.C.), horizontale (A.H.) ou oblique (A.O.).

Diverses observations et mesures sont faites au cours de la végétation sur les ramifications latérales des plants (longueur et nombre des nœuds); l'analyse de la croissance n'a porté que sur les pousses latérales des zones arquées distales (Z.A.D.) et proximale (Z.A.P.), la zone hors arcure n'ayant pratiquement pas eu de pousses.

En fin juin, la réaction à l'arcure, dans la Z.A.P., est de même ordre pour les 3 traitements, avec toutefois un plus grand étalement de la répartition des pousses vigoureuses pour les traitements A.H. et A.O. Dans la Z.A.D., les pousses vigoureuses sont de moins en moins nombreuses dans l'ordre des traitements A.O., A.H., A.C.

En fin de végétation, la réaction dans la Z.A.P. présente un caractère uniforme. Les pousses vigoureuses se concentrent au niveau des nœuds, les plus proches du sommet de l'arc pour le traitement A.C., alors qu'elles s'étalent de cette zone de réaction vers les nœuds de la Z.A.D., les plus voisins du sommet de l'arc, pour les autres traitements. Dans la Z.A.D., seul le traitement A.O. suscite au moins une pousse très vigoureuse, les autres traitements ne formant pratiquement, dans cette zone, que des pousses de vigueur moyenne.

L'élaboration du modèle de réaction final se produit en deux temps; d'une part, une réaction d'élongation très localisée au printemps et, d'autre part, une reprise d'activité de rosettes et pousses courtes après la fin juin.

Colette Bernard-Dagan, G. Illy, Ginette Pauly (I.N.R.A., Station de Recherches forestières, 33 - Bordeaux).

VARIATION DES MONOTERPÈnES CHEZ LE PIN MARITIME. ETUdE DE DIVERS ClONES

Les essences présentes dans les rameaux de Pin Maritime développés au cours de la saison précédente ont une constitution différente dans le bois et dans les tissus extérieurs au bois: l'essence du bois renferme presqu'exclusivement de l' $\alpha$-pinène et du $\beta$-pinène ; celle des tissus extérieurs au bois (parenchyme cortical et liber) contient, outre les pinènes $\alpha$ et $\beta$, du $\Delta 3$-carène, du myrcène et du limonène.

A l'intérieur de la provenance landaise, une étude des arbres, individu par individu, a toujours révélé la présence de ces monoter i'nes dans les divers tissus, mais en des proportions très variables d'un individu à l'autre, lo taux de chaque monoterpène étant calculé par rapport à 100 de monoterpènes totaux.

A l'intérieur des clones, dans tous les cas examinés, la composition monoterpénique des essences de «l'arbre mère» se retrouve très fidèlement chez les greffes qui en sont issues; certains arbres mères avaient jusqu'à 85 ans et les greffes avaient été faites au maximum 9 ans auparavant sur des porte-greffes âgés de deux ans.

Ainsi un clóne peut être caractérisé non seulement par le rapport $\alpha$-pinène/ $\beta$-pinène dans les essences du bois, mais aussi par l'abondance particulière (ou l'absence quasi totale), du $\Delta 3$-carène, ou du myrcène, ou du limonène dans les tissus extérieurs au bois. Ces caractères sont indépendants de l'époque à laquelle est pratiquée l'analyse. 
Cependant, cette identité s'accompagne toujours chez les greffes d'une légère élévation $\mathrm{du}$ taux relatif de $\beta$-pinène dans l'essence du bois et des taux de $\Delta 3$-carène, myrcène ou limonène dans l'essence des tissus extérieurs au bois. Cette remarque paraît traduire un caractère plus «jeune» des tissus qui synthétisent les essences, chez les greffes par rapport aux arbres mères.

Ce travail fera l'objet d'une publication dans les Annales des Sciences forestières.

F. Piton (Service du Professeur Schaeverbecque, 122, rue Cuvier, 75 - -Paris $\left(5^{\circ}\right)$

Aperçu de Quelques travaux de cytologie sur des tissus D'arbres Cultivés «in vitro » AU laboratoire de biologie végétale de Fontainebleau.

\section{F. Barnoud, $\mathbf{M}^{\mathrm{me}}$ Mollard et $\mathbf{M}^{\mathrm{mec}}$ Fustache.}

ETUde COMPARATIVE SUR LE MÉTABolisme dU TISSU CAMBIAL DES SOUCHES EN CULTURE.

G. Illy et B. Lemoine (I.N.R.A., Station de Recherches forestières, 33 - Bordeaux).

DENSITÉ DE PEUPLEMENT, CONCURRENCE ET COOPÉRATION CHEZ LE PIN MARITIME.

L'article complet a déjà fait l'objet d'une publication, dans le numéro 2, de 1970, des Annales des Sciences forestières. 